\title{
Spontaneous Closure of Atrial Septal Defects in Premature vs Full-Term Neonates
}

\author{
T. Riggs, ${ }^{1}$ S.E. Sharp, ${ }^{2, *}$ D. Batton, ${ }^{1}$ M.E. Hussey, ${ }^{1}$ E. Weinhouse ${ }^{1}$ \\ ${ }^{1}$ Departments of Pediatrics, Divisions of Cardiology and Neonatology, William Beaumont Hospital, 3535 W. 13 Mile Road, \\ Suite 747, Royal Oak, MI 48073, USA \\ ${ }^{2}$ University of Michigan Medical School, Ann Arbor, MI 48109, USA
}

\begin{abstract}
Our hypotheses were that the following factors influenced closure of atrial septal defects (ASDs) detected in neonates: estimated gestational age (EGA), the presence of a persistent ductus arteriosus (PDA), severity of pulmonary disease, gender, and the initial size of the ASD. Our population consisted of 82 newborns (38 premature and 44 term) who were found before the age of 1 month to have an ASD. Closure of ASDs was analyzed using both Kaplan-Meier survival analysis and the Cox proportional hazards model, each with the five covariates. The hazard ratio (or ratio of instantaneous closure rates) of term vs preterm infants was $3.60(95 \%$ CI = 1.80-7.20), whereas the hazard ratio for infants with a PDA (compared to infants with no PDA) was 2.41 (95\% $\mathrm{CI}=1.28-4.54)$. Multivariate analysis showed that each of these terms (PDA and EGA) were independent predictors of ASD closure. The hazard ratio of ASD closure for each of four levels of pulmonary disease was 0.632 [95\% CI $=0.453-0.881]$. We conclude that the majority of neonatal ASDs will close, with EGA and PDA acting as important influences on closure.
\end{abstract}

Key words: Atrial septal defects - Persistent ductus arteriosus — Prematurity

The existence of a fetal interatrial channel has been known since the time of Galen [10]. This connection, the foramen ovale, is bounded by the limbus of the fossa ovalis (septum secundum) superiorly and to the right and by the septum primum inferiorly and to the left. In the fetus, this flap-like arrangement allows unidirectional, right-to-left interatrial flow through the foramen ovale, maintaining an important channel of the fetal circulation. Postnatally, with increased pulmonary blood flow and

Correspondence to: T. Riggs

*Second-year medical student increased flow into the left atrium, the left atrium's pressure exceeds that of the right atrium and the foramen ovale functionally closes. This functional closure eventually leads to anatomic closure in that fibrous adhesions permanently close the atrial septum in most individuals, with about $20 \%$ of adults having a patent foramen ovale $[8,11]$.

Previous studies have documented the postnatal timing of spontaneous closure of atrial septal defects (ASDs), particularly in term infants [4, 12]. In this study, we examined the relationship between the time course of closure of ASDs and covariates such as gender, estimated gestational age (EGA), severity of pulmonary disease, the presence of a persistent ductus arteriosus (PDA), and the initial size of the ASD. We believe that this is the first multivariate analysis of these factors, allowing us to suggest mechanisms for closure or persistent patency of neonatal ASDs.

\section{Patients and Methods}

We reviewed our medical records and identified 82 newborn infants ( 38 premature and 44 term) who were found to have an interatrial shunt by color Doppler examination before the age of 1 month. All patients had follow-up echocardiographic exams. We included neonates with a PDA, peripheral pulmonic stenosis, or tricuspid insufficiency but excluded all patients with other significant heart malformations.

We performed complete two-dimensional (2-D) and color Doppler examinations using either a 5.0- or a 7.5-MHz transducer (HewlettParkard HP-1500). We measured the diameter of the atrial defect from a subcostal four-chamber view, using either the 2-D echocardiogram or the color Doppler exam, following the method described by Radzik et al. [12] in that the primary tool was the 2-D echocardiogram, whereas the color Doppler exam was used in those cases (usually smaller defects) in which the ASD was not well delineated by 2-D echocardiography alone. Patients were classified into three groups based on the initial size of the atrial defect: group I $(\leq 3 \mathrm{~mm})$, group II ( $3 \mathrm{~mm}<$ ASD $<5 \mathrm{~mm})$, and group III $(5 \mathrm{~mm} \leq \mathrm{ASD} \leq 7 \mathrm{~mm})$ [5]. If a PDA was present at the time of the initial examination, then infants were classified as having a PDA. 
Table 1. Gestational age and ASD size categories

\begin{tabular}{llll}
\hline & $\begin{array}{l}\text { Group I } \\
(\text { ASD } \leq 3 \mathrm{~mm})\end{array}$ & $\begin{array}{l}\text { Group II } \\
(3 \mathrm{~mm}< \\
\text { ASD }<5 \mathrm{~mm})\end{array}$ & $\begin{array}{l}\text { Group III } \\
(5 \mathrm{~mm} \leq \\
\text { ASD } \leq 7 \mathrm{~mm})\end{array}$ \\
\hline Preterm & 9 & 13 & 16 \\
Term & 14 & 16 & 14 \\
\hline
\end{tabular}

On the basis of the clinical history, we divided the infants into four categories by the degree of respiratory distress: (I) term infants with no or transient respiratory distress; (II) preterm infants with no or minimal respiratory distress, including tachypnea or increased oxygen requirement that persisted for several days but excluding mechanical ventilator support or use of exogenous surfactant; (III) preterm infants who required mechanical ventilation for $<1$ month, oxygen supplementation for $<1$ month, or use of exogenous surfactant; and (IV) preterm infants who required mechanical ventilation for $\geq 1$ month and/or developed radiological evidence of bronchopulmonary dysplasia.

We used several tests and methods in our statistical analysis. A Kaplan-Meier survival function was used to produce a graphic representation of the closure of the ASDs as a function of time. A loglikelihood ratio test was used for comparison of curves generated by different strata of a covariate. The Cox proportional hazards model ("ties" were handled using the "exact" method) was used to calculate the hazard ratios for strata of the covariates. A significance level of $p$ $<0.05$ was chosen for all hypothesis testing. For statistical analysis, we used the computer software SAS 6.12 (The SAS Institute) and S-Plus (Mathsoft). This study was reviewed and approved by our hospital review board.

\section{Results}

There were 38 premature infants, with a mean gestational age of $31 \pm 4$ weeks (range 23-36.7 weeks). The remaining 44 infants were term, with a mean gestational age of $39 \pm 1$ weeks (range 37-42 weeks). Eighteen of 38 premature infants $(47 \%)$ and 30 of 44 term infants $(68 \%)$ were female.

For preterm infants, the mean age at diagnosis was $11 \pm 9$ days (range 1-26) and the mean follow-up was $450 \pm 530$ days. For term infants, the mean age at diagnosis was $3 \pm 5$ days (range 1-26) and the mean followup was $135 \pm 190$ days. There was a mean of three studies done per patient (range 2-11).

There were 23 infants in group I and 29 infants in group II, and the remaining 30 infants were in group III. Their allocation by gestational age is shown in Table 1 . Among the term neonates, 26/44 (59\%) had a PDA, whereas among the preterm infants $13 / 38(34 \%)$ had a PDA at their initial study (Table 2).

There were 44 term infants with no or minimal respiratory distress (class 1), 17 preterm infants with no or mild respiratory distress (class 2), 8 preterm infants with moderate respiratory distress (class 3 ), and 13 preterm infants with severe, chronic respiratory distress (class 4 ) (Table 3).
Table 2. Gestational age and PDA status

\begin{tabular}{lll}
\hline & PDA present & PDA closed \\
\hline Preterm $^{a}$ & 13 & 25 \\
Term $^{b}$ & 26 & 18 \\
\hline
\end{tabular}

${ }^{a}$ Mean age at diagnosis $=11$ days.

${ }^{b}$ Mean age at diagnosis $=3$ days.

Table 3. Pulmonary status and gestational age

\begin{tabular}{lccc}
\hline EGA & \multicolumn{2}{c}{ Weeks } & \\
\cline { 2 - 4 } & $23-29$ & $29-32$ & $32-37$ \\
\hline Class II & 0 & $4(2)^{a}$ & $13(3)$ \\
Class III & $2(1)$ & $3(2)$ & $3(0)$ \\
Class IV & $11(5)$ & $2(0)$ & 0
\end{tabular}

${ }^{a}$ Numbers in parentheses represent the number of patients who had a PDA at their initial examination.

\section{Univariate Comparisons}

The closure times were significantly different $(p<$ 0.0001) for the preterm vs term infants (Fig. 1). The median closure time for the preterm infants was 752 days (95\% CI $=353-1747$ days), whereas the median closure time for term infants was 119 days $(95 \% \mathrm{CI}=85-253$ days).

The closure times were also significantly different $(p$ $<0.009$ ) for the PDA vs non-PDA infants (Fig. 2). The median closure time for the PDA infants was 103 days (95\% CI $=48-255$ days), whereas the median closure time for non-PDA infants was 752 days $(95 \%$ CI $=$ 353-1373 days) (Table 4).

There was a significant effect of the degree of pulmonary disease on ASD closure. For each increment increase in severity status from class 1 to class 4, the hazard ratio for ASD closure decreased by a factor of 0.632 $(95 \%$ CI $=0.453-0.881)$.

There was no significant effect of gender on closure time of ASDs $(p=0.44)$. There was only a weakly significant effect of ASD size on the closure rate $(p=$ 0.22). Median closure times by initial size of ASD were as follows: group I, 370 days; group II, 136 days; group III, 501 days $(p=0.081)$. However, using a binary variable for the initial size of the ASD, namely class A defined as $<5 \mathrm{~mm}$ and class $B$ defined as $\geq 5 \mathrm{~mm}$, there was a significant effect of ASD size on closure $(p=$ $0.038)$. If the initial size of the ASD $(<5 \mathrm{~mm}$ vs $\geq 5$ $\mathrm{mm})$ was examined separately for the term vs preterm neonates, there was no significant difference found ( $p=$ 0.44 and $p=0.09$, respectively). 

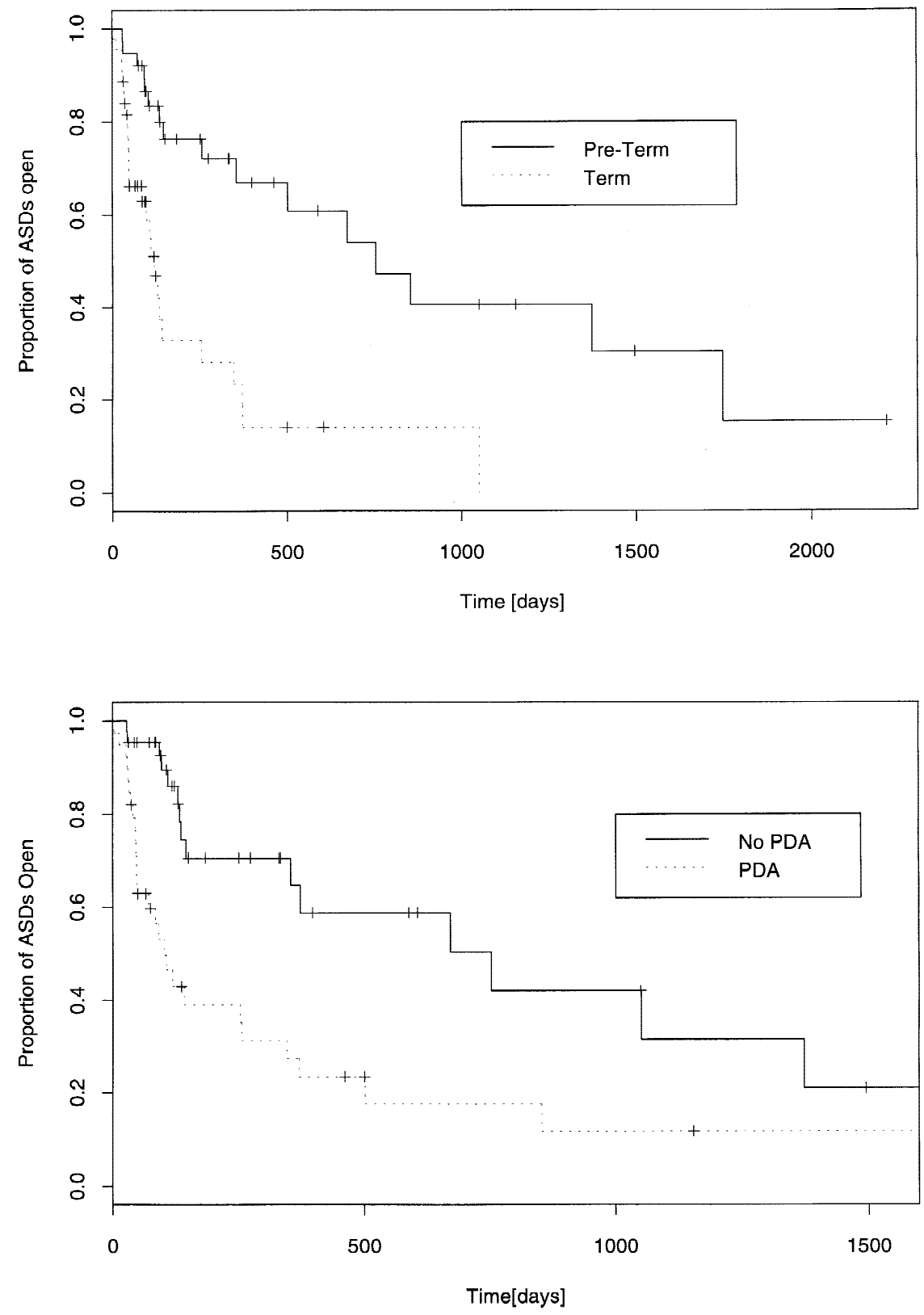

Fig. 1. The Kaplan-Meier survivor functions for preterm vs term infants showing earlier ASD closure for the term infants. The two curves are significantly different; log likelihood ratio test; chi-square $=$ 25.785; $1 \mathrm{df} ; p<0.0001$.
Fig. 2. The Kaplan-Meier survivor functions for PDA vs non-PDA infants showing earlier closure for the PDA infants. The two curves are significantly different; log likelihood ratio test; chi-square $=$ 10.9659; $1 \mathrm{df} ; p<0.0009$.

\section{Multivariate Comparisons}

When the closure times for the four strata (term with PDA, term without PDA, preterm with PDA, and preterm without PDA) were examined, the trend was very significant $(p<0.0001)$ (Fig. 3). The median times to closure in ascending order were term with PDA (48 days), term without PDA (134 days), preterm with PDA (501 days), and preterm without PDA (752 days).

For the Cox proportional hazards model, we first constructed a full model with covariates: gender, gestational age (term vs preterm), class of pulmonary disease
Table 4. Median closure times

\begin{tabular}{lc}
\hline & Days \\
\hline Preterm & 752 \\
Term & 119 \\
PDA present & 103 \\
PDA closed & 752 \\
Term with PDA & 48 \\
Term without PDA & 134 \\
Preterm with PDA & 501 \\
Preterm without PDA & 752 \\
\hline
\end{tabular}




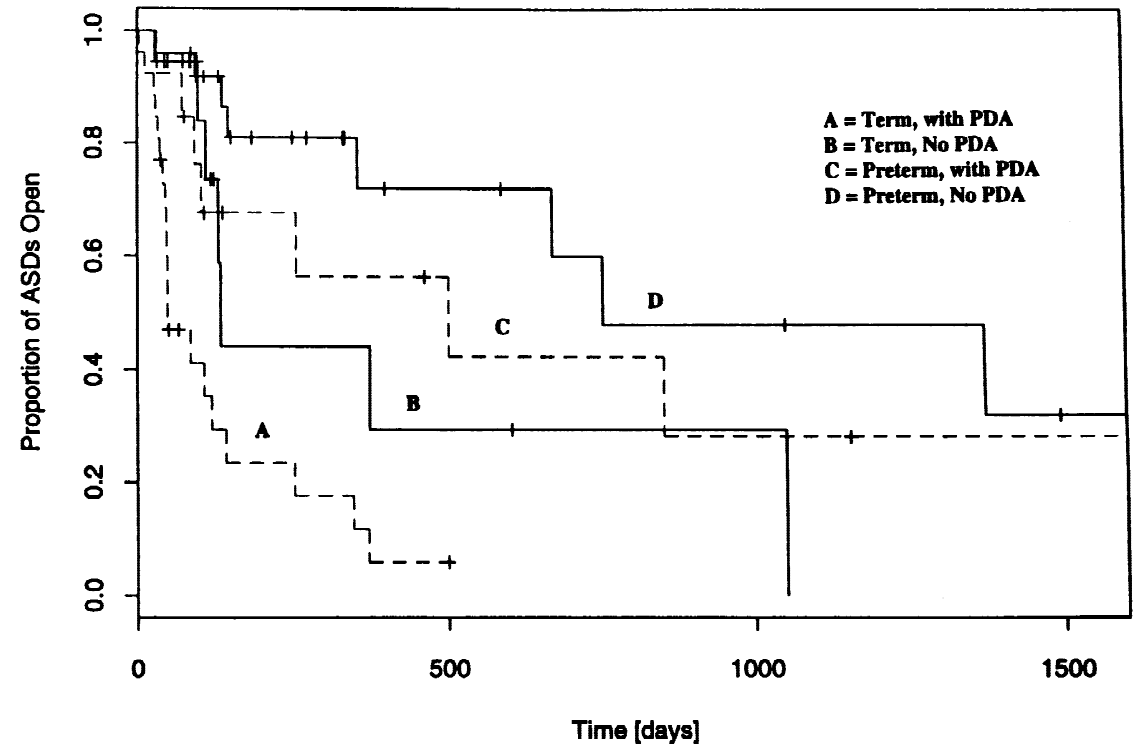

Fig. 3. The Kaplan-Meier survivor functions for the four strata of preterm vs term and PDA vs non-PDA categories. Although there is some overlap of the curves, the median increases as preterm and term are compared. Within each EGA class, the median increases as PDA is compared with non-PDA. The four curves are significantly different; $\log$ likelihood ratio test; chi-square $=36.2054 ; 3 \mathrm{df} ; p<0.0001$ severity, initial ASD size, and initial presence of a PDA. Gender and initial ASD size (whether a binary, threelevel, or continuous variable) could be dropped from the model without any loss of significance, whereas both gestational age and PDA were significant and independent predictors of ASD closure. Given the close association between EGA and pulmonary status and the sample size in the various classes of pulmonary status, it was not possible to separate their influence on ASD closure. However, the best predictive model used PDA status and EGA status, each as a binary variable. This best model was significantly better than a model using PDA and pulmonary class, chi-square $=5.316,1 \mathrm{df}$, and $p=$ 0.012 .

The hazard ratios (with regard to likelihood of ASD closure) were as follows: "pre-term with no PDA" was the baseline (hazard rate defined as $=1.00$ ) comparison group. The "pre-term with PDA" group had a hazard ratio of $2.42(95 \% \mathrm{CI}=1.28-4.54)$, whereas the "term with no PDA" group's ratio was $3.60(95 \% \mathrm{CI}=1.80$ 7.20). For the "term with PDA" group, the hazard ratio is multiplicative; thus, it is 8.69 (95\% CI $=3.14-24.1)$.

\section{Interactions Among Variables}

We examined the effect of many interactions, particularly those between the initial size of the ASD and term vs preterm status ( $p=0.4035)$ and between PDA status and degree of pulmonary disease $(p=0.3124)$.

\section{Discussion}

A left-to-right shunt via an atrial defect in a neonate presents a problem in nomenclature (i.e., a patent fora- men ovale vs an atrial septal defect). On the one hand, most of these defects will resolve during infancy; on the other hand, some represent secundum atrial septal defects which will persist and ultimately require repair. This differentiation cannot be made on the basis of the initial size alone because large defects with evidence of right ventricular volume overload and even congestive heart failure initially may close spontaneously $[2,6,9]$. We chose to call these defects ASDs as a generic term without further classification as a true secundum ASD. Since most ASDs found in neonates will spontaneously close, our study cannot be extrapolated to the natural history of secundum ASDs found in older infants or children.

Fukazawa et al. [4] examined 102 consecutive neonates (the majority were term infants) and found that $24 \%$ had an ASD in the first week, $13 \%$ of these were still present by 1 week to 1 month, 7\% were present after 1 month, 5\% were present after 6 months, and $2 \%$ were present by age 1 year. These findings are consistent with our study since we began with a population of neonates with an ASD and found that, months to years later, most of these had closed. Radzik et al. [12] presented their findings in a study similar in format to ours; they examined 101 infants found at age $<3$ months to have an ASD, in which $87 \%$ spontaneously closed. In their study, the size of the defect was associated with timing of closure. There is no mention in their study of whether PDAs were also present. No specific mention was made in their study of whether any of their patients were preterm, but all patients were asymptomatic, excluding many preterm infants. Ghisla et al. [6] described 29 infants under 12 months of age with significant left-to-right atrial shunts, of whom 14\% had spontaneous closure by 30 months. 
Cockerham et al. [2] described serial cardiac catheterization data showing ASD closure in 15 of 87 patients [17\%] who had an initial age ranging from neonates to 4 years. Using cardiac catheterization data, Mahoney et al. [9] described spontaneous closure of ASDs in 10 infants and children ( 8 term and 2 premature) out of an original group of 24 , or $42 \%$ of those infants who had presented at $<18$ months.

We believe our study is unique in that we compared term with preterm infants and compared multiple variables with the outcome of ASD closure. In this way we were able to show the strong influence of the presence of a PDA and gestational age on ASD closure.

In our study, the initial size of the ASD had only a weak influence on the subsequent closure of the ASD, which could be made significant only if we compared the largest ASD size category with the remainder of the patients. Most of the atrial defects in this study were not large. If our study group included patients who had large secundum ASDs (very few of whom would be detected as neonates), then the initial ASD size would certainly have an effect on subsequent closure. Similarly, there is a female: male incidence of secundum ASDs of about $2: 1$. In our population, there was neither a clear gender difference in prevalence of ASDs nor a detectable gender difference in closure rates of ASDs.

\section{Influence of PDA}

The presence of a PDA at the time of initial diagnosis of an ASD was strongly associated with earlier closure of the ASD. This influence was present in both term and preterm infants. Our group of term infants were examined for the first time, on average, at age 3 days. This is a time when the majority of term infants would be expected to have a closed ductus arteriosus. The presence of a PDA at age 3 days would be expected to increase pulmonary venous return to the left atrium, increase left atrial pressure, and therefore hasten closure of the ASD. Term infants with a PDA had a median time of ASD closure of less than half that of the infants without a PDA (48 vs 134 days). Among the patients in this study with a PDA, there was eventually universal spontaneous PDA closure, and the PDAs were usually small at the time of the initial examination. The effect of a large, persistent PDA might be different in that the enhanced blood flow to the left atrium could be sufficiently large so as to enlarge the left atrium and separate the edges of the atrial opening. This could potentially delay or prevent the closure of an ASD. However, among our patients, those with a PDA were much more likely to have earlier closure of their ASD compared to those without a PDA.
In the preterm infants in this study, the presence of a PDA was also found to have a significant effect on ASD closure. The age at initial diagnosis was, on average, 11 days. This is an age at which many PDAs have already closed in all but the most premature or most seriously ill premature infants. Therefore, our cohort of premature infants is unusual in that the majority have already had closure of their PDAs. Although the effect of PDA on ASD closure was weaker for the preterm infants, it was qualitatively the same in that preterm infants with a PDA had a median ASD closure of 501 days, whereas the preterm infants without a PDA had a median ASD closure of 752 days. The same argument regarding the influence of the PDA on left atrial pressure is true, but the opposing right atrial pressure would be expected to be higher, given the likelihood of pulmonary disease.

\section{Influence of Prematurity}

Premature infants, particularly those with gestational age from 24 to 29 weeks, frequently have chronic pulmonary disease, are more likely to require ventilatory support, and frequently have some residual amount of labile pulmonary hypertension that persists for months [1, 7]. These factors could contribute to poor right ventricular compliance and elevated right atrial pressure, which would tend to keep the flap of the foramen ovale open. Furthermore, poorer lung compliance in premature infants could lead to greater phasic respiratory changes in intrathoracic, left atrial, and right atrial pressures. The effect would be a less stationary position of the interatrial septum, perhaps preventing or delaying closure.

\section{Statistical Analysis}

The analytical approach we used is a standard one used to build a predictive model. Although individual analysis of each independent variable is useful, often a multivariate approach provides more insight, particularly if one allows for interaction among the variables. In this way, one can construct the most efficient model for predicting outcome based on the most economical use of the independent variables [3]. The theory behind this is based on the concept of the log-likelihood ratio, in which the fit of the data is compared to its log-likelihood and the model with the greatest likelihood is the best.

In this case, we first constructed a "full model" with all five covariates, including various logical interaction terms. In stepwise fashion, one drops the variables from the model and assesses the impact of the loss of each 
variable on the predictive ability of the model. Another useful technique is to examine a variable as continuous, binary, or multiple-strata data. Our final analysis and most parsimonious model included PDA (as a binary variable) and term vs preterm (also as a binary variable). All other terms and interactions did not add significantly to the model. For example, it might be argued that the absolute size of the ASD should be indexed for the various somatic sizes encountered from 23 to 42 weeks. The data do not support the hypothesis that initial ASD size or interaction of ASD size with EGA add to the model.

\section{Limitations of the Study}

This study was entirely noninvasive. Without direct measurements of left atrial and right atrial pressures, one cannot absolutely confirm our arguments about the effects of the presence of a PDA on left atrial pressure or of the influence of prematurity on right atrial pressures. Although this is a large sample of term and preterm infants, a larger sample including more preterm infants having mild, moderate, or severe pulmonary disease could elucidate whether it was prematurity or pulmonary disease, or an interaction between them, that led to delayed ASD closure. Similarly, a larger sample of preterm infants would have included more infants with large PDAs, which might have helped in the evaluation of the influence of a PDA on ASD closure.

\section{References}

1. Berman W, Yabek SM, Dillon T, Burstein R, Corlew S (1982) Evaluation of infants with bronchopulmonary dysplasia using cardiac catheterization. Pediatrics 70:708-712

2. Cockerham JT, Martin TC, Gutierrez FR, et al. (1983) Spontaneous closure of secundum atrial septal defect in infants and young children. Am J Cardiol 52:1267-1271

3. Collett D (1994) Modelling survival data in medical research. Chapman \& Hall, New York, pp 53-106

4. Fukazawa M, Fukushige J, Ueda K (1988) Atrial septal defects in neonates with reference to spontaneous closure. Am Heart J 116: $123-127$

5. Gentile R, Stevenson G, Dooley T, et al. (1981) Pulsed Doppler echocardiographic determination of time of ductal closure in normal newborn infants. J Pediatr 98:443-448

6. Ghisla RP, Hannon DW, Meyer RA, Kaplan S (1985) Spontaneous closure of isolated secundum atrial septal defects in infants: an echocardiographic study. Am Heart J 109:1327-1333

7. Goodman G, Perkin RM, Anas NG, et al. (1988) Pulmonary hypertension in infants with bronchopulmonary dysplasia. $J$ Pediatr 112:67-72

8. Hagen PT, Scholz DG, Edwards WD (1988) Incidence and size of patent foramen ovale during the first 10 decades of life: an autopsy study of 965 normal hearts. Mayo Clinic Proc 59:17-20

9. Mahoney LT, Truesdell SC, Krzmarzick TR, Lauer RM (1986) Atrial septal defects that present in infancy. Arch Dis Child 140: $1115-1118$

10. Patten BM (1931) The closure of the foramen ovale. Am J Anat 48:19-44

11. Patten BM (1938) Developmental defects at the foramen ovale. Am J Pathol 14:135-161

12. Radzik D, Davignon A, van Doesburg N, et al. (1993) Predictive factors for spontaneous closure of atrial septal defects diagnosed in the first 3 months of life. J Am Coll Cardiol 22:851-853 